\title{
The photoreceptor mosaic
}

\begin{abstract}
The organisation of the human photoreceptor mosaic reflects evolutionary strategies for optimising visual information under a wide range of stimulus conditions: (1) The rod population dominates (max. $170000 / \mathrm{mm}^{2}$ at c. $30^{\circ}$ sup.) except for the central $2^{\circ}$ and along the ora serrata. (2) Density of cone inner/outer segments reaches up to $300000 \mathrm{~mm}^{2}$ in the fovea. A bundle of $c$. 300-500 foveolar cones are further distinguished by having their synaptic terminals located within the capillary-free zone. Radial displacement $(>350$ $\mu \mathrm{m})$ of foveal cone terminals may result in the lesion of two sets of cone pathways by perifoveal laser treatment. Along the ora serrata peripheral cone density (c. 4000) rises within a small rim $\left(1^{\circ}\right)$ to up to 20000 , but may be considerably decreased by cystoid degenerations. For the L- and M-cone subpopulations ratios of 2:1 to $1: 1$ and random arrangement are suggested. (3) Blue-sensitive (S-) cones constitute a regular and independent submosaic of $c .7 \%$ across the periphery. An annular maximum (1000-5000/ $\mathrm{mm}^{2}$ ) at $c .1^{\circ}$ surrounds the foveola. There density decreases and irregular zones lacking $\mathrm{S}$-cones result in tritan deficiencies.
\end{abstract}

Key words Cones, Fovea, Laser coagulation, Peripheral cystoid degeneration, Photoreceptor, Retina, Rods, Topography

The photoreceptor mosaic is the primary sampling matrix of the human visual system, limiting the bandwidth of the information flow. ${ }^{1}$ Each location of the retinal projection plane is characterised by a varying interplay of input to three major subsystems for maximising either sensitivity or spatial and colour discrimination. Information is fed into these subsystems (which are not always and everywhere separated) by rods, L-/M-cones and $\mathrm{S} /$ cones. Despite the importance of these data for, for example, differentiation of normal state and variability from (early) pathological alterations, surprisingly few papers (e.g. Yamada, ${ }^{2}$ Misotten, ${ }^{3}$ Adams et al. ${ }^{4}$ ) have followed the classical works of $\varnothing_{\text {sterberg }}^{5}$ and Polyak $^{6}$ on the cone mosaic. Recently a wealth of new data on the photoreceptor mosaic organisation has become available, in particular as a result of the development of techniques and criteria for specific labelling of cone subtypes, the refinement of psychophysical and microspectrophotometrical methods, and more systematic studies on topography and development (see Curcio and Hendrickson ${ }^{7}$ ). The present overview will try to give a short update and include some specific observations on the fovea and extreme periphery that may be of particular relevance for ophthalmologists.

The original data presented are from retinas of eyes obtained for corneal transplants (see Pum et al. ${ }^{8}$ for details). Cone photopigment antibodies were kindly provided by J. Nathans. ${ }^{9}$

\section{General morphology of photoreceptors}

Although proportions may be extremely variable, all mammalian retinas studied have been found to have a dual set of photoreceptors: rods and cones (Fig. 1). Besides having specific response kinetics ${ }^{10}$ and sensitivity, rods and cones also differ in their metabolic ${ }^{11}$ and morphological characteristics.

Rods have thin tubular inner and outer segments, the latter protruding furthest into the pigment epithelium (Fig. 1a). The stack of rod photopigment membrane discs is separated by a continuous cell membrane from extracellular space. Rod terminals (spherules) contain only one to three triadic synapses. Rod spherules are connected to adjacent cone pedicles by punctate junctions ${ }^{12}$ but not to neighbouring rods.

The term 'cones' is derived from the conical shape of the inner segments in mid-peripheral cells (Fig. 1b). It may provide a larger entrance pupil at the external limiting membrane for guiding directed light towards the outer segments (Fig. 1a, right focus plane). However, the conical tapering is not a consistent morphological feature of this cell type. A comparison of cones along a meridian of human retina shows considerable variation. ${ }^{13}$ The elongated foveal cones ${ }^{14,15}$ have considerably smaller diameters $(c .1 .3 \mu \mathrm{m})$ than peripheral rods $(c .2 .5 \mu \mathrm{m})$. Cones close to the ora ${ }^{16}$ on the other hand have small, bent inner and outer segments (Fig. 10). More consistent criteria for the differentiation of cones are identifiable at fine structural level: basal outer segment disc membranes are open to the extracellular space, the inner segments contain highly packed mitochondria, and the synaptic terminal
P.K. Ahnelt

Department of General and Comparative Physiology Medical School

University of Vienna

Vienna

Austria

P.K. Ahnelt

Inst. f. allg. \& Vergl.

Physiologie

Univ. Wien

Schwarzspanierst. 17

A-1090 Vienna

Austria

e-mail:

peter.ahnelt@univie.ac.at Web: http://www.univie.ac. atNergl-Physiologie/www/ gphy_morph.html 

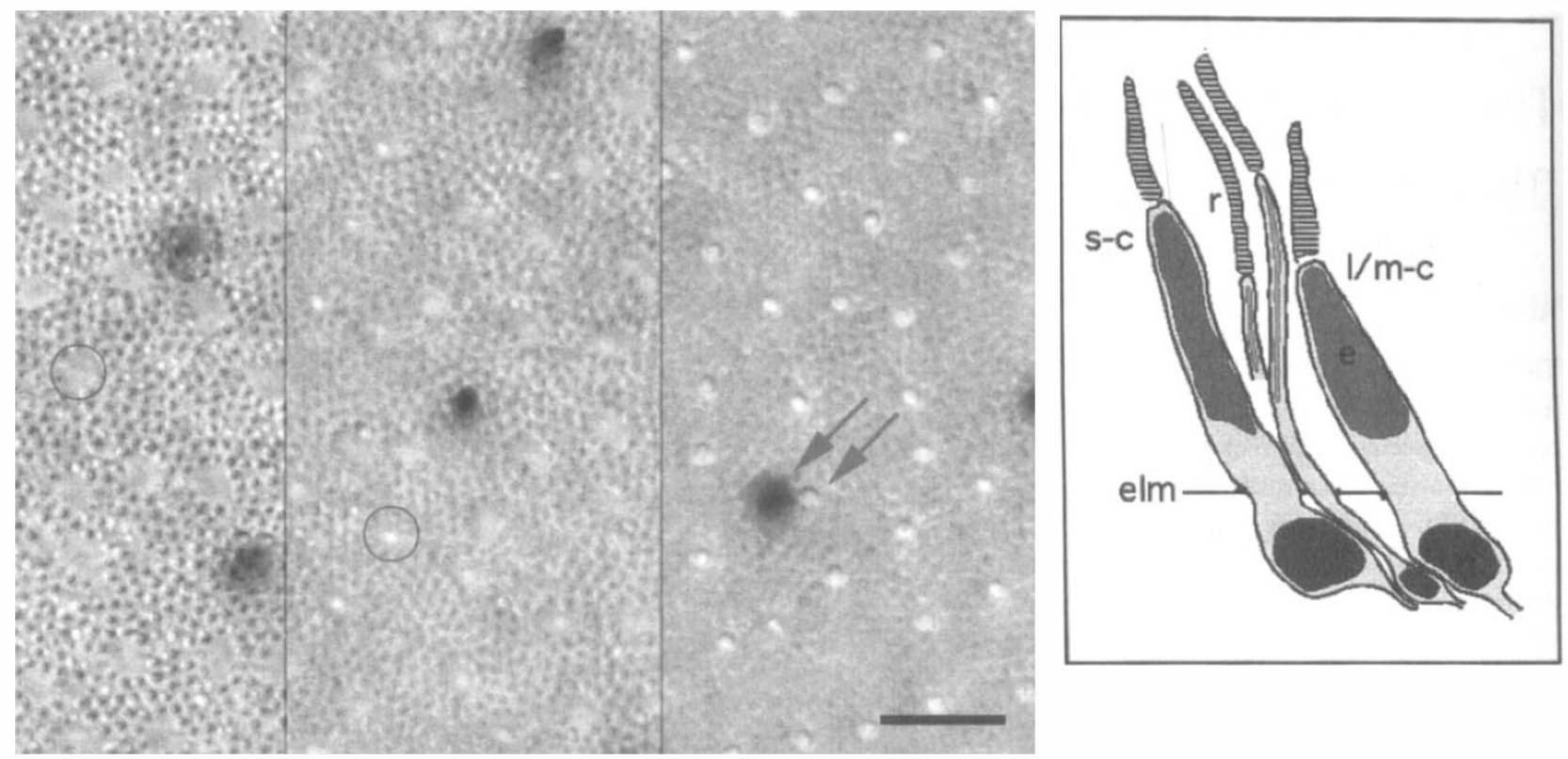

a

b

Fig. 1. Human peripheral photoreceptors. (a) Retinal wholemount with immunocytochemical labelling (JH 455) for S-cone photopigments. DIC digital micrograph. Three focus steps at consecutive level of photoreceptor outer segments. Left: Empty spaces defined by more proximal cone inner segments are surrounded by tips of several rows of rod outer segments. Centre: Focus plane includes the longer tips of labelled S-cone outer segments. Some L-/M-cones are identifiable by the light-guide effects of their tips (circle). Right: Focus plane includes all cone outer segments plus distal rod inner segments. Note the regular arrangement of both $S$ - and L/M-cones but accidental apposition of an S-cone to an $M / L-c o n e$ (double arrow) implying independent organisation of chromatic submosaics. Scale bar represents $50 \mu \mathrm{m}$. (b) Schematic diagram of three morphological photoreceptor types. s-c, S-cone; $1 / m-c$, long or middle wavelength sensitive cone; $r$, rod; elm, external limiting membrane with adherent junctions to surrounding Müller cell processes (not shown).

(pedicle) is expanded to carry the increased number of connections, ${ }^{12,17}$ establishing dozens of superficial and invaginating synapses to interneurons ${ }^{18}$ and to neighbouring photoreceptors. ${ }^{19}$

It has long been demonstrated psychophysically ${ }^{20}$ that three cone types with specific photopigments must exist to create the chromatic antagonisms of what we now call the modern and ancient (dichromate) colour system. ${ }^{21}$ Specific L- and M-cones representing the base for the second ('modern') chromatic subsystem providing us with trichromacy have evolved rather recently among primates. ${ }^{22}$ So far no morphological criteria for direct differentiation of $\mathrm{L}$ - and $\mathrm{M}$-cones have been reported but there may be differing connectivities along their midget pathways. ${ }^{23}$ Characterisation by

microspectrophotometry ${ }^{24}$ and photopigment transmittance imaging ${ }^{25}$ of primate mosaic samples suggests a random distribution of $\mathrm{M}$ - and L-cones at ratios of $c$. 1:1. Consequently no selectivity seems to be present at the level of mutual telodendritic connections ${ }^{12}$ among L-/M-pedicles (Fig. 9), although specific modulation of these electrotonic junctions remains a possibility.

More progress has been possible in our understanding of the photoreceptoral origins of 'blue/ yellow' pathways. We can now histochemically, morphologically and immunocytochemically differentiate S-cones by subtle but distinctive criteria. S-cones differ from L- and M-cones ${ }^{26,27}$ by their slightly longer, less conical inner segment (Fig. 1a,b). S-cones may be specifically identified by mitochondrial formazan precipitation ${ }^{28}$ after specific chromatic stimulation and NBT incubation, by selective uptake of Procion dyes injected into the vitreous, ${ }^{29,30}$ by lack of carbonic anhydrase labelling ${ }^{31}$ or by low reactivity for NADPHdiaphorase. ${ }^{32}$ Reliable labelling of spectral subpopulations, including those from human postmortem material, has become possible by the development of anti-photopigment antibodies (AB) by Szél et al. ${ }^{33}{ }^{33}$ erea $^{27}$ and Chiu and Nathans. ${ }^{9}$

Reconstruction of S-cone synaptic pedicles ${ }^{12,34}$ has revealed structural differences likely to be of functional significance for primary colour processing. S-cones lack telodendritic connections to neighbouring cones almost completely, in contrast to the network between M- and L-cones. Thus direct modulation of S-cones appears possible only via rods and indeed linear summation for rod and S-cone signals has been reported at threshold. ${ }^{35}$ Recently specific chromatic connectivity patterns to underlying horizontal cells could be identified at light and electron microscopical level ${ }^{36}$ and were confirmed by intracellular labelling and recording. ${ }^{37}$ The identification of a specific type of bipolar cells ${ }^{38}$ also contributed to the continuing attempts to track elements of colour-related retinal circuitry.

\section{Topographies}

Attributing altered states of retinal function to specific losses at the receptoral level requires a detailed knowledge of the general topographies of the receptor subtypes. Systematic studies, in particular by Curcio and 


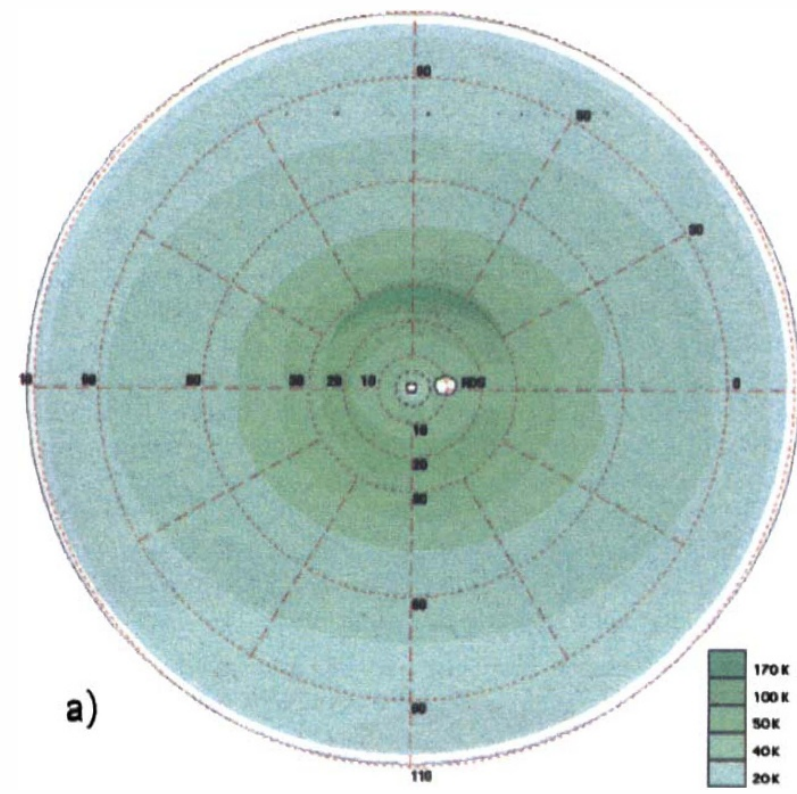

Fig. 2. Schematic topographies of three retinal photoreceptor subpopulations. Perimetric overlay indicates eccentricities and meridians in degrees. White spot represents papilla lacking all distal retinal elements including PR. (a) Rods occur in most retinal locations except the foveola (diameter c. $250 \mu \mathrm{m}$ ) and along a small rim at the ora serrata. (b) L/M-cone. A steep foveal peak is surrounded by an elliptical central area extending nasally; there is a cone-enriched rim at the ora serrata. (c) S-cones. Minimum in the foveola, maximum 1500-5000 cones $/ \mathrm{mm}^{2}$ in the foveal ring $\left(c .1^{\circ}\right)$. Very low values $(<10 \%)$ in the periphery.

collaborators, ${ }^{39}$ have provided improved data. The schematic diagrams of Fig. 2 describe the general topographies of the three photoreceptor mosaics.

\section{Rod topography}

In contrast to the retinas of most other vertebrates the characteristic feature of most mammalian photoreceptor sets is the extensive rod population. The human retina is a typical example with the exception of a small specialisation - the fovea. Fig. 2a shows a schematic topography of rods in human retinas. Under scotopic conditions the fovea represents a second 'blind spot'. Its anatomical correlate - the rod-free zone - has been found to extend across the central $250-350 \mu \mathrm{m},{ }^{26,27}$ representing c. $1^{\circ}$ of visual field. From there rod densities rise steeply, approaching $160000-190000$ at 4-6 $\mathrm{mm}\left(20-30^{\circ}\right)$ with a 'hot spot' in the superior meridian. ${ }^{7}$ This number can be seen to match the peak foveal cone densities in some
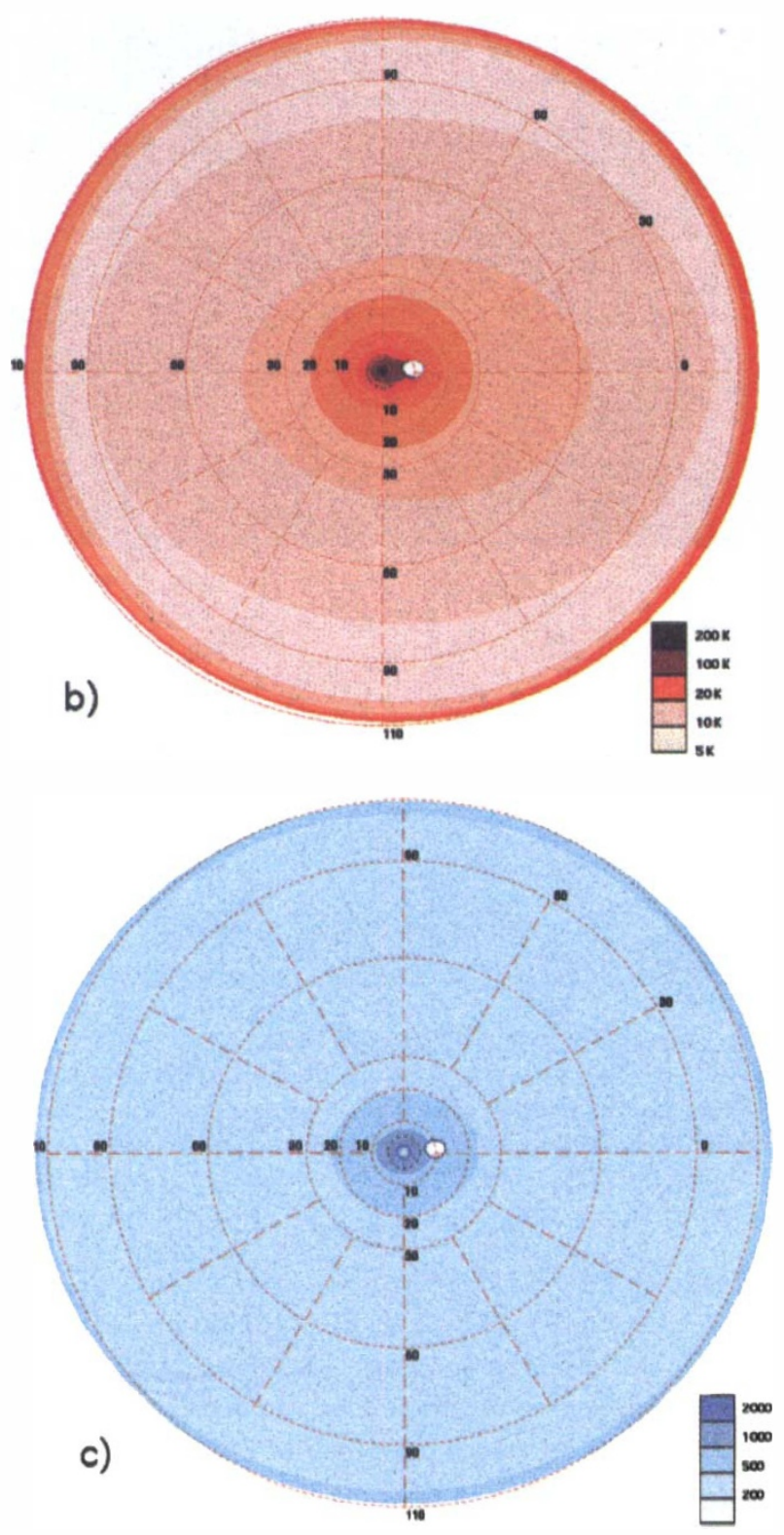

adult retinas (see below), but the concentration of rods mainly serves scotopic sensitivity via rhodopsin accumulation and signal convergence. As revealed by detailed topographic mapping ${ }^{7}$ the distribution of human rods reflects the interference with the cone system. The ring of rod peak density is reciprocal to the isodensity lines of the cone system by being compressed elliptically and indented along the horizontal meridian. At further eccentricities the numbers decrease but are more than compensated by the increase in rod diameters. Coverage of retinal area reaches $65 \%$ in peripheral retina.

\section{Cones}

Across most of the retinal surface the cones constitute a small proportion of photoreceptor cells (c. $4000 / \mathrm{mm}^{2}$ ), with overall coverage of $c$. $20 \%$. More than $90 \%$ of the cones are $\mathrm{L}$ - and $\mathrm{M}$-cones. By forming a regular mosaic they determine the maximum sampling (Nyquist) 

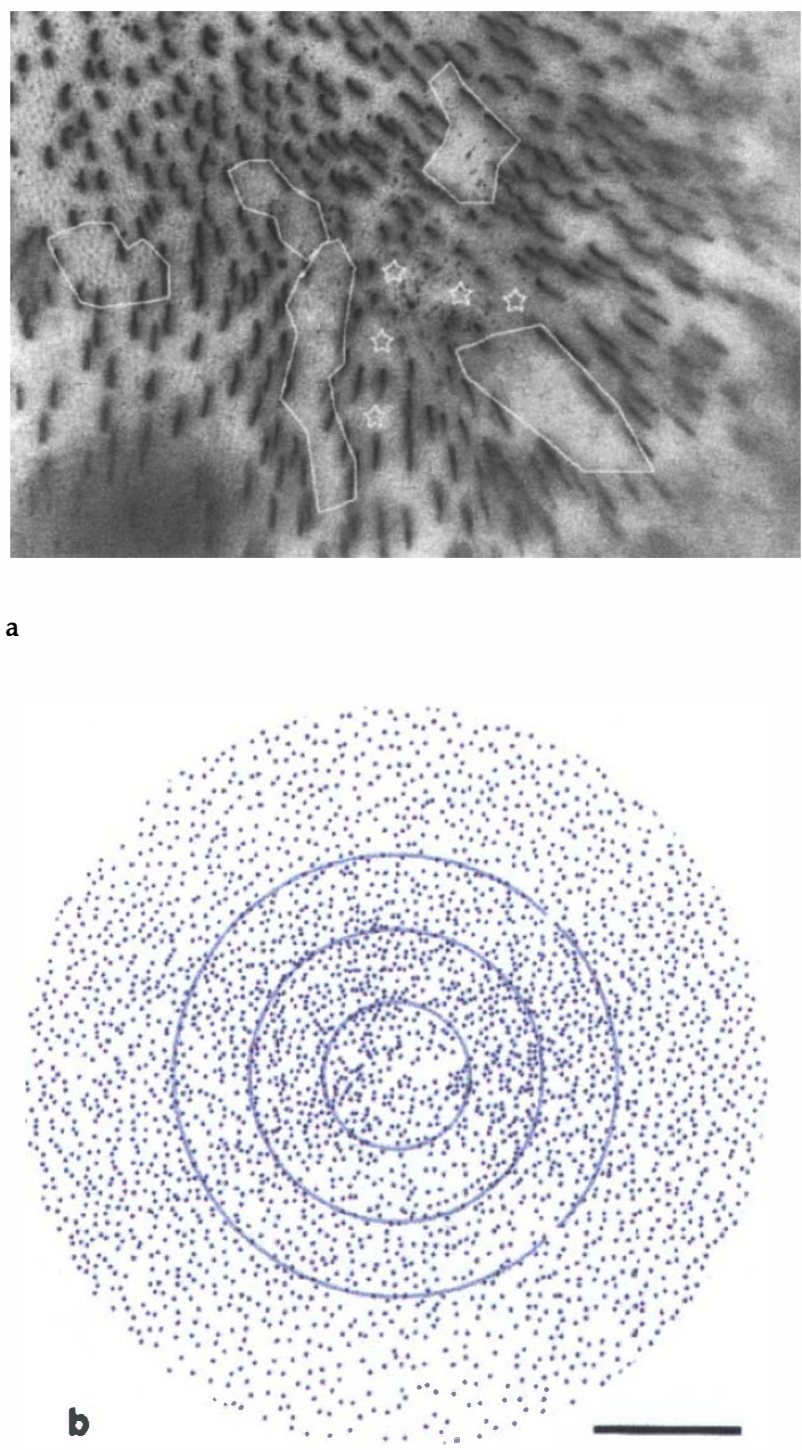

frequency for visual resolution at high-contrast conditions. Densities and cone coverage are $40 \%$ lower in the temporal retina than in the nasal retina.

\section{S-cone topography}

Recently various techniques for direct identification of S-cones have become available. Histochemical markers are available ${ }^{28,31,32}$ high-affinity dyes such as Procion Yellow $^{29,30}$ may be taken up selectively by S-cones, morphological criteria ${ }^{12}$ allow $S$-cones to be distinguished at the inner segments (Fig. 1) or at the synaptic pedicles, ${ }^{26,33}$ and recently anti-photopigment antibodies ${ }^{9,27,33}$ have allowed selective mapping of the outer segments. The optical quality of the shortwavelength image components is poor due to scattering and chromatic aberration. Sampling high spatial and temporal frequencies has favoured the dominance of $\mathrm{L} / \mathrm{M}$-cones at the available lattice positions. S-cones represent less than $10 \%$ of the cone population and only $1-2 \%$ of the available retina area is dedicated to the detection of short-wavelength light. An accumulation of
Fig. 3. S-cone distribution in the fovea (59-year-old woman) labelled with S-cone anti-photopigment antibody. (a) Foveolar S-cones. Digital micrograph with focus projection to compensate for the slope of the outer fovea. Small pigment epithelial granules demarcate a central depression in the outer fovea. (b) Plot of S-cone locations in the foveal centre (diameter of sample $=1 \mathrm{~mm}$ ). Concentric rings are arranged around the maximum $M / L$-cone densities at intervals of $100 \mu \mathrm{m}$.

(c) False colour map of S-cone density from (a). The ring of maximum density (c. $1300 / \mathrm{mm}^{2}$ ) at c. $200 \mu \mathrm{m}$ is incomplete and compressed vertically. In this retina the interpolation procedure does indicate a depression but not a complete lack in the centre.

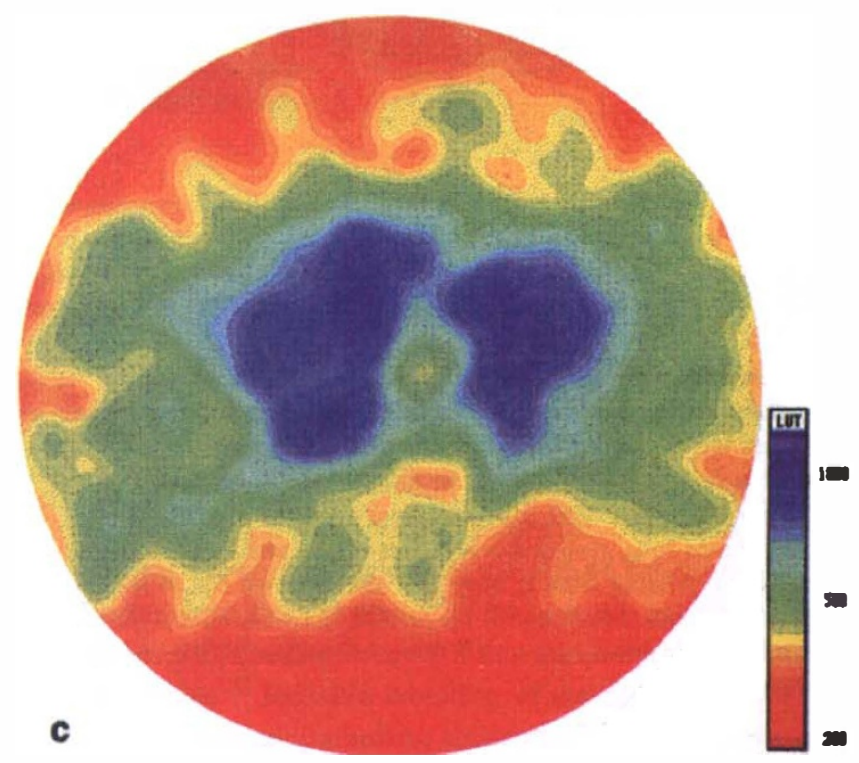

S-cones is found around the foveola (Fig. 4). S-cones reach c. $2000 / \mathrm{mm}^{2}$ in this perifoveolar ring (c. $1^{\circ}$ ecc.), a mere fourfold increase compared with the periphery, while the foveolar peak of L/M-cones may be more than 40 times the peripheral values. Since the observations by König $^{40}$ and the rediscovery of the phenomenon by Willmer ${ }^{41,42}$ it is known that the foveal centre is insensitive to blue light. Recent psychophysical studies attribute this foveal tritanopia (or tritanomaly) to a decrease in or lack of S-cones. The scarcity of S-cones is clearly detectable in studies on S-cone resolution ${ }^{43,44}$ but not in our normal visual experience. Efficient amplification and interpolation of the S-cone signal is already performed within the retina, possibly including specific interactions with the rod system. ${ }^{35}$ There is basic agreement about the existence of a foveolar S-cone density minimum, but reports suggest either a relative or complete lack in the center. Fetal Rhesus monkey appear to have a continuous S-cone mosaic in the primordial fovea. ${ }^{45}$ In the adult monkey ${ }^{33}$ as well as in samples of human foveae ${ }^{27}$ some central lattice locations are found empty, suggesting a specific loss (apoptosis?) of some foveolar S-cones during development. From our own 


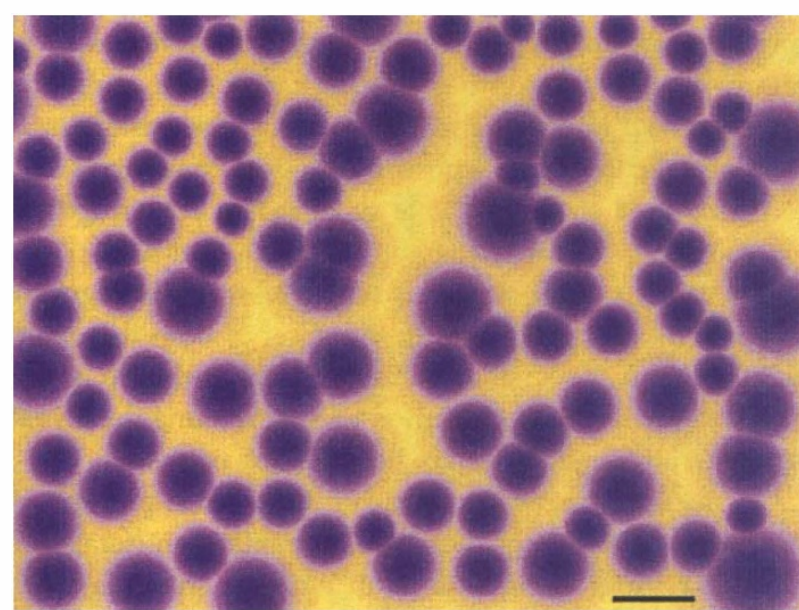

Fig. 4. False colour map of S-cone sensitivity obtained from a foveal semithin section of Rhesus fovea ${ }^{33}$ by Voronoi tessellation of S-cone positions and subsequent overlay of the difference of two (blue-yellow) Gaussians. These receptive domains associated with individual S-cones cover an irregular central area. Some isolated patches remain uncovered and are likely to be tritanopic. Scale bar represents $20 \mu \mathrm{m}$.

limited number of samples it seems that a proportion of the discrepancies may be based on individual variation. We have seen centres with a diameter of $c .100 \mu \mathrm{m}$ free of labelled S-cones but also cases where the pure L/M-cone patches do not include the centre (Fig. $3 a, b)$. The result is an individual landscape of foveal blue sensitivity (Fig. 3c) that may include a central tritan area (Fig. 4).

\section{Increased cone density at extreme peripheral retina}

An interesting phenomenon, which was described early ${ }^{46}$ but went largely unnoticed, is a second zone of increased cone density in the extreme periphery. ${ }^{16}$ At a distance of c. $2 \mathrm{~mm}$ from ora serrata cone density starts to rise again, in particular along the nasal rim. The peculiar shape of photoreceptors in these regions makes it difficult to distinguish cell types morphologically. Antiphotopigment labelling (Fig. 5), however, confirms that cones are the dominant population and that they have well-developed outer segments. Cones finally outnumber rods within a rim of $c .400 \mu \mathrm{m}$ along the ora serrrata. When the effective light-collecting areas represented by summed cone and rod inner segment cross-sectional areas are considered the dominance of the cone system is even more pronounced (factors of 5-10). This striking non-linearly of the cone mosaic raises questions about its developmental ${ }^{47}$ and functional significance, in particular for stimulus detection along the peripheral border of the visual field. ${ }^{48}$

\section{Degeneration in the extreme periphery}

Cystoid and reticular degeneration is an alteration ${ }^{49,50}$ of most adult retinas with unclear aetiology, ${ }^{51}$ and therefore is included here as a quasi-general feature of the retinal mosaic.

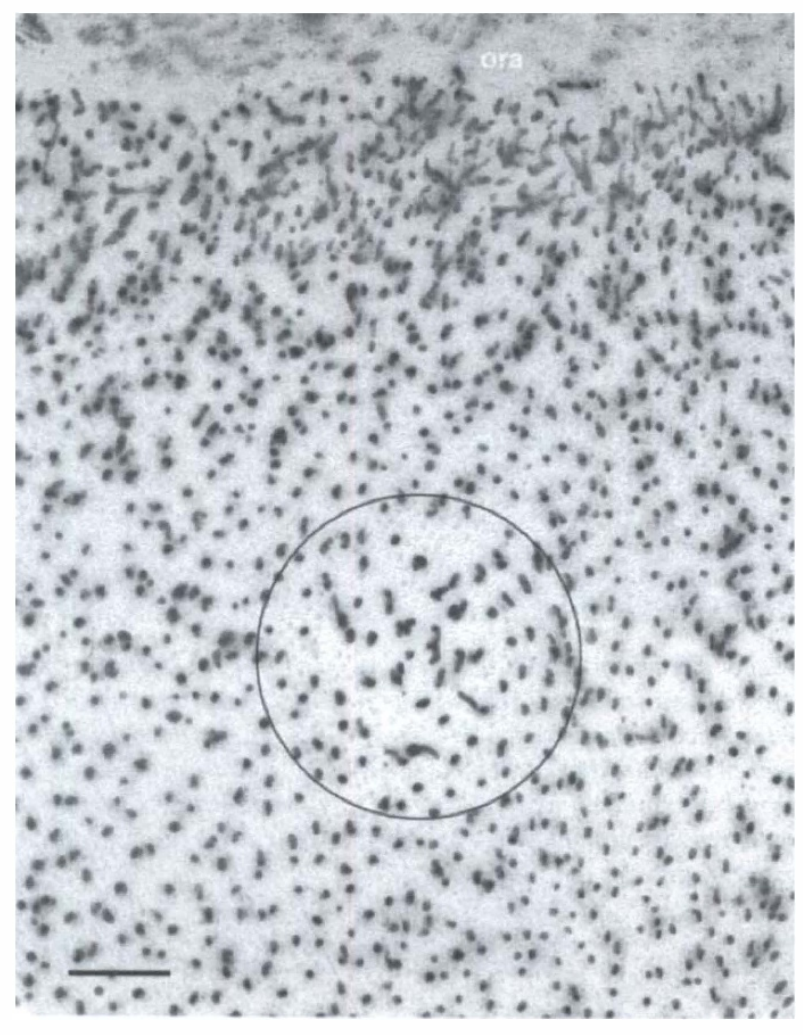

Fig. 5. Cones at the ora serrata (18-year-old man). Digital micrograph obtained from projection of a stacked focus series. Cone outer segments (JH 455 labelling) increase steeply at the ora (top). A small collapsed cyst (circle) carries a diminished number cones. Scale bar represents $100 \mu \mathrm{m}$.

The cystoid spaces occurring mostly in the inner nuclear and outer plexiform layer are bordered by meandering ridges of continuous retina. ${ }^{52}$ This remaining tissue has been found to consist mainly of interneurons and Müller cells. It may be expected that cystoid degenerations alter the overlying receptor mosaic, although the external limiting membrane is preserved even in advanced stages. Labelling with antiphotopigment antibody allows convenient evaluation of receptor density. Small isolated cysts (Fig. 5, circle) may represent initial degenerative stages with receptors remaining largely intact. A sample with advanced cystoid degeneration in Fig. 6, however, shows a patchy cone pattern when labelled for L/M-cone photopigment. The extension of the particular sample is related to the course of a capillary belonging to the array of terminal vascular cascades. The overlay of $\mathrm{M} / \mathrm{L}$-cone positions

(Fig. 6, right) confirms severe receptor loss (>50\%) above the cystoid spaces. Further progression leads to almost complete loss of immunoreactive photoreceptor elements.

Thus while an intriguing rise in cone density can be found along the ora serrata (see above) the actual increase in (photopic) sensitivity in larger zones of this region may often be more than compensated by degeneration. More systematic studies will be needed to understand the role of this border zone in the intact or degenerating state. 


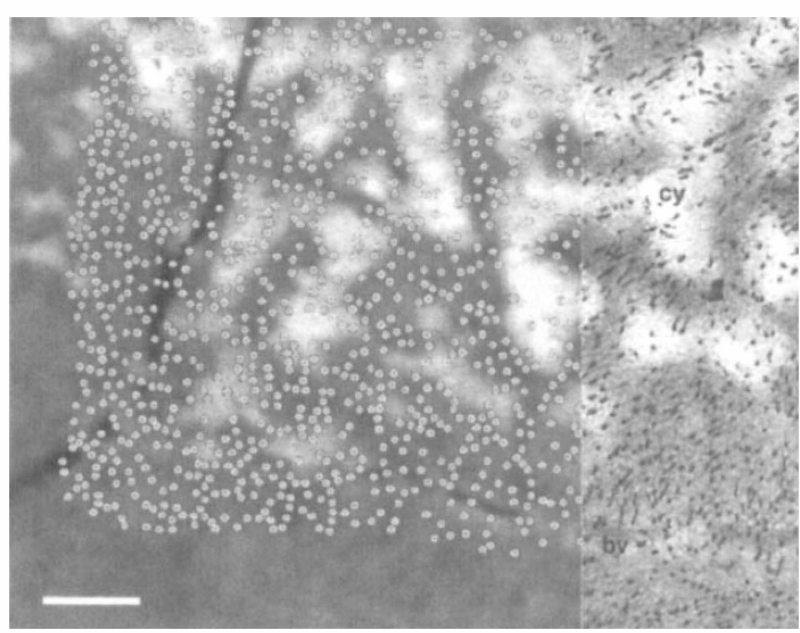

Fig. 6. Peripheral cystic degeneration. Three adjacent segments from temporal ora serrata (47-year-old man). Right: Focus on extensive intraretinal cystoid spaces (cy) located within a triangular region defined by bifurcation of a terminal vessel (bv) and the ora (beyond top right-hand corner of image). Left: Plotting the positions of labelled cones reveals substantial loss of cones $(\geqslant 70 \%)$ above the cysts. Scale bar represents $50 \mu \mathrm{m}$.

\section{Fovea}

The image elements projected on the fovea centralis - the small area (diameter c. $1.5 \mathrm{~mm}, 5^{\circ}$ ) around the eye's optical axis - are exploited by multiple channels along the central visual pathways. Topograpically the fovea is situated within the area centralis (diameter $6 \mathrm{~mm} \approx 15^{\circ}$, 'posterior pole') characterised histologically by at least two layers of ganglion cells. Within the fovea the position of the innermost capillary ring demarcates the foveal avascular zone (FAZ, c. 500-600 $\mu \mathrm{m} \approx 2^{\circ}$ ). This ophthalmoscopic landmark is usually within a zone of decreasing thickness: the foveal slope (Fig. 7). It results from the radial displacement of most neural elements from a flatter foveal floor (diameter c. $200 \mu \mathrm{m}$ ).

The foveal cones are characterised by maximum condensation of the inner and outer segments and the considerable displacement between these sensory elements and the synaptic connections to interneurons. ${ }^{53}$ At the distal receptoral pole the inner and outer segments increase in length, and because the curvature of the pigment epithelium remains unaltered this creates a depression of the external limiting membrane or outer fovea. In flat tangential sections (Fig. 8) this results in a sequential concentric exposure of foveal cone levels.

Combined studies of cone spacing and photopigment densitometry of the living eye $\mathrm{e}^{54}$ suggest that the elongation of outer segments may preserve the pigment volume by compensating the thinning of both the outer segment and the smaller entrance pupil at the tip of the inner segment. Thus resolution increases along the foveal slope while sensitivity may be roughly constant.

A pure cone area but at lower densities is present already at the time of receptor cell generation (fetal days). The high density in adult retina is largely a consequence of subsequent centripetal condensation, ${ }^{\overline{3}}$ a process which may persevere during childhood and possibly into adolescence. Data on foveal cone maxima are still limited and vary greatly between adult individuals. ${ }^{7,8,26,29}$ Both deviate substantially. The value of c. $167000 / \mathrm{mm}^{2}$ (Østerberg ${ }^{5}$ ) from a 16-year-old retina, still cited as reference in many diagrams and textbooks of physiology and ophthalmology, is well below the maximal value of $>320000 / \mathrm{mm}^{2}$ reported recently. This variability may account in part for the spatial acuity variation in normal eyes. The steepness of the foveal peak and the adjacent cone-dominant region is not symmetrical but maintains higher values along the nasal meridian. The elevated levels extend to form a small collar of higher density around the papilla.

\section{The inner retinal layers are extremely diluted at the foveal floor but not entirely absent}

Labelling of proximal cellular elements of S-cones allows the specialised projection pattern (Fig. 9) for minimising the absorption and scattering of light to be revealed. The elongation of foveal cone axons (fibres of Henle) allows the proximal layers to be displaced radially and arranged concentrically around the foveal floor. Beyond the central zone a concentric ring of displaced pedicles establishes a regular mosaic linked by telodendria and is situated above the innermost rings of capillaries (Fig. 10). The amount of displacement varies. For monkey retinas

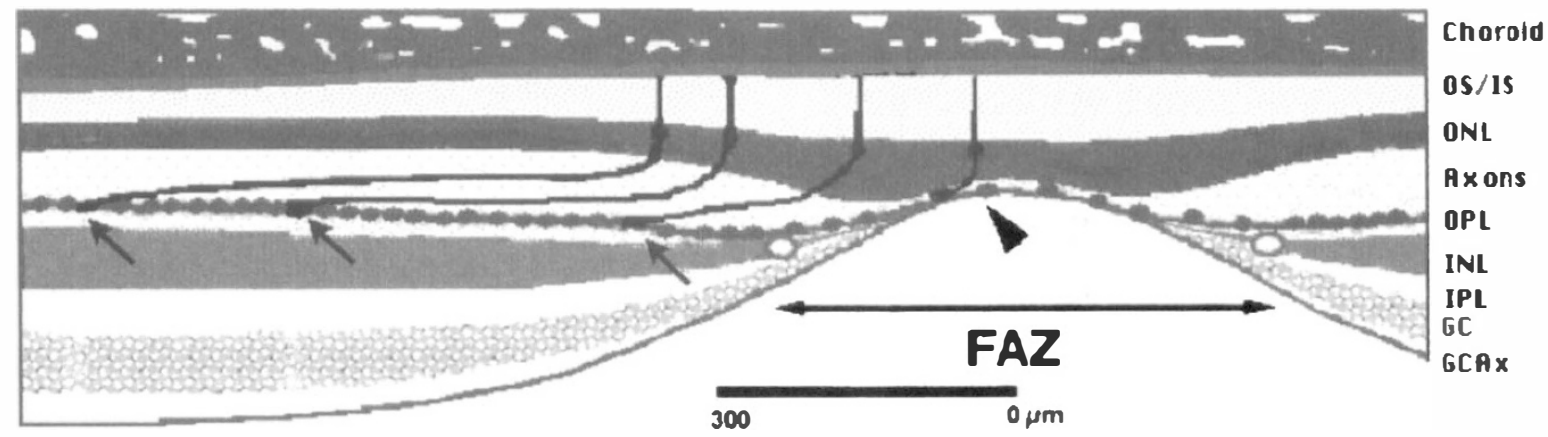

Fig. 7. Foveal radial microarchitecture (schematic diagram modified from Polyak). ${ }^{6}$ The foveal floor lacks the continuous layers of proximal retina since most elements are displaced radially (arrows) as indicated by some cones and interneurons (black). The extent of displacement of con' synaptic terminals (pedicles) varies depending on outer segment location. The radial shear of proximal layers implies a dual effect of local laser treatment: affected tissue may include a set of outer cone elements from one set as well as displaced pedicles/axons from a second set of cones. 


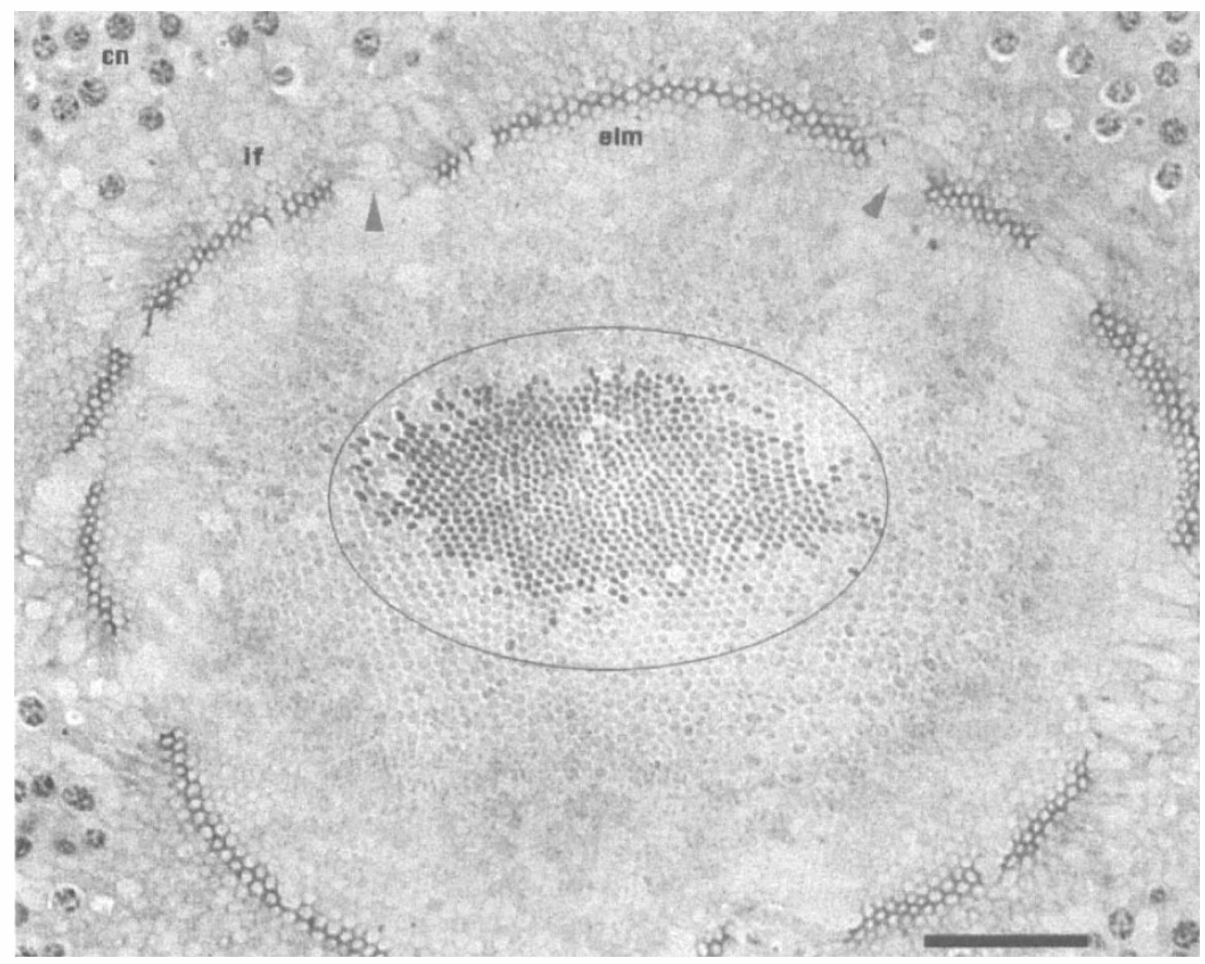

Fig. 8. Foveal cone mosaic (19-year-old man). Semithin tangential section, $1 \mu m$; Toluidine Blue, maximum density $141000 / m m^{2}$. Due to the outer foveal slope a sequence of levels is visible. Ellipse: Central bundle of cones (c. 500) shows darker staining of inner segments, indicating a specific post-mortem change. Along the external limiting membrane (elm) the inner segment becomes less distinct due to protruding Müller cell microvilli. The ring of tight junctions of the elm is incomplete (arrowheads). Beyond the elm are cone inner fibres (if) and cell bodies (cn). Scale bar represents $50 \mu \mathrm{m}$.

maximum values of $300 \mu \mathrm{m}$ have been described; ${ }^{56}$ for Golgi-labelled human retinas maximum values of $c$. $400 \mu \mathrm{m}$ have been found (Ahnelt and Kolb, unpublished). The foveal floor is usually considered to be filled by specialised Müller cells and to lack neuronal elements. However, results from methods such as labelling ganglion cells (retrogradely) ${ }^{57}$ or the dopaminergic plexus ${ }^{58}$ suggest that a dispersed population of interneurons and an associated plexus are present even in the foveola. Serial sections at light and electron microscope level ${ }^{59}$ reveal that there are a number of cone pedicles at the foveal floor in the foveal avascular zone (Fig. 11). The axons of these cones descend at steep angles and terminate amongst some other neurons dispersed there (Fig. 11). They originate in a small bundle of axons descending almost vertically (Fig. 7, arrowhead). Electron microscopic sections and serial reconstruction of the foveal floor reveal that OPL processes converge to the proximal surface of such pedicles establishing a local 'knot' of OPL. ${ }^{59}$ These pedicles terminate within the FAZ and are not organised in regular arrays. Their number (c. 300-400) approximately matches the bundle of central cones distinguishable at the inner segment level (Fig. 8). It seems likely that these cones function under specific metabolic conditions since their proximal elements are not reached directly by intraretinal vasculature as is the case for peripheral cones. Recently evidence is arising that foveal cones express a specific molecular and metabolic identity. ${ }^{60}$

At the outer segment level the rod-free zone (diameter c. $250 \mu \mathrm{m}$ ) of the foveal photoreceptor mosaic displays a highly organised crystalline organisation (Fig. 8) providing a basis for optimised spatial sampling. ${ }^{61}$

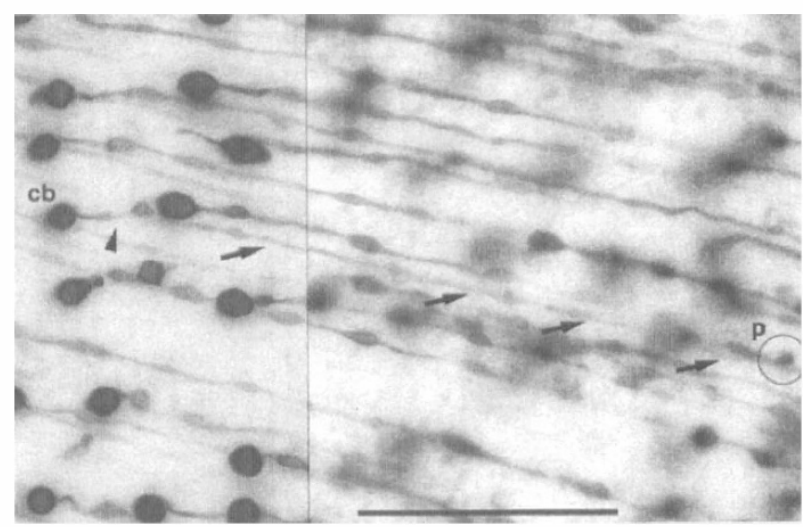

Fig. 9. Course of cone of fibres of Henle. Focused projection extracted from a z-series of digital micrographs from cell bodies to pedicles. Since only the sparse S-cones are labelled by JH 455 individual cells may be traced along their entire length (here, c. $300 \mu \mathrm{m}$ ). Fibres of Henle first descend from the cell body (arrowhead) through the all-cone outer nuclear layer and then take a straight course (arrows) with bead-like extensions towards a small pedicle (circle). Scale bar represents $100 \mu \mathrm{m}$. 


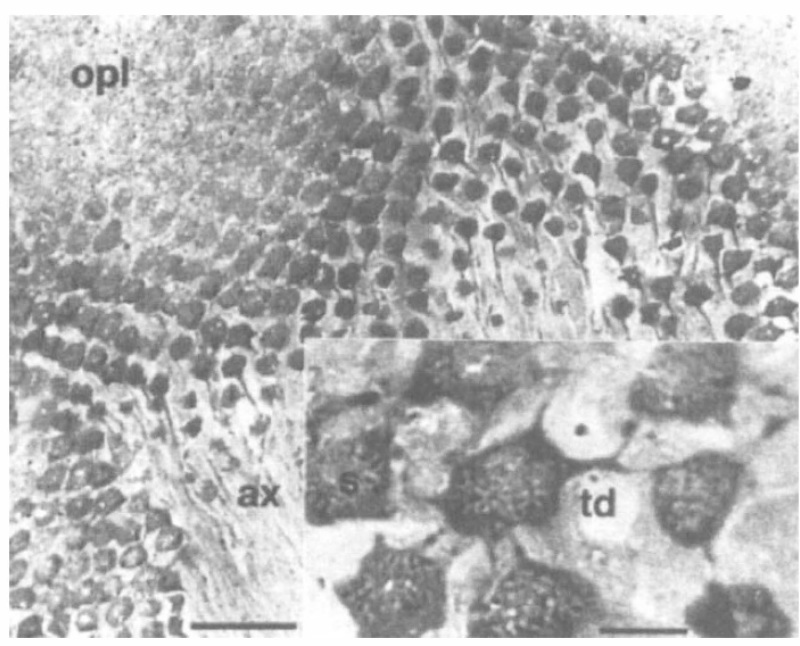

Fig. 10. Pedicles of foveal cones situated in the vascular zone (37-yearold man). Tangential section, $1 \mu \mathrm{m}$; Stevenel's Blue staining. This single layer of pedicles is organised in densely packed regular arrays corresponding to the mosaic of the more centrally situated inner/outer segments. Insert: The proximal pedicle surface carries numerous invaginating and superficial synapses (s). In addition an extensive network of telodendrites $(t d)$ connects most cones, establishing a network of gap junctions. Scale bars represent $50 \mu \mathrm{m}$ (left) and $10 \mu \mathrm{m}$ (right).

Detailed analysis, however, demonstrates the presence of lattice defects distributed non-randomly. Cone positions with $</>6$ neighbours tend to accumulate in the central bundle or are arranged in linear series subdividing the hexagonal mosaic in a series of patches with varying axial orientation. ${ }^{8,62}$ This subdivides the mosaic into patches with similar crystalline orientation, possibly reorganising and relaxing the lattice from shearing stress accumulating during inward migration and condensation. Analogous to the less regular positions of $\mathrm{S}$-cones in peripheral retina (Fig. 1a) S-cones are more frequently associated with these lattice defects. Image sampling across this lattice at the Nyquist limit may

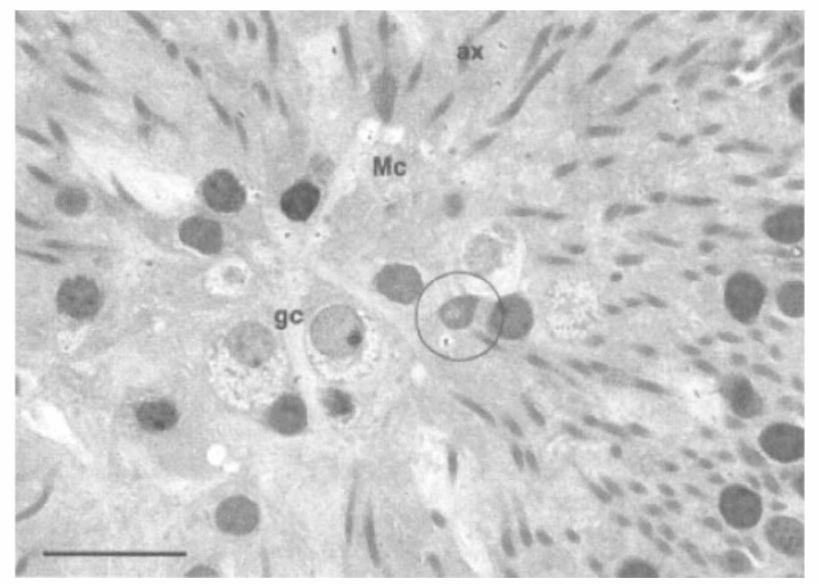

Fig. 11. Foveal floor. Semithin section from series of Fig. 8. The centre is mostly filled by lightly stained voluminous Müller cell (Mc) elements. gc, two ganglion cells and other neurons; circle, isolated cone pedicle; ax, darkly stained axons (fibres of Henle) of other cones. Scale bar represents $50 \mu \mathrm{m}$. reduce aliasing seen as 'zebra stripe' effects, ${ }^{63,64}$ and can be useful for evaluating the foveal mosaic parameters in the living eye. ${ }^{65}$

\section{Centrifugal displacement of foveal cone pedicles}

Foveal development starts from a 'hot spot' of all-cone mosaic in the developing retina. ${ }^{66}$ The subsequent elongation of foveal cone axons has important implications for the treatment of various pathological conditions affecting the retina, vasculature or choroid by punctate laser coagulation. In general strategies are chosen that minimise damage of the fixation point. The inner ring of capillaries is useful as a guiding landmark to set, for example, $100 \mu \mathrm{m}$ laser burns at no less than $200 \mu \mathrm{m}$ eccentricity. However, as pointed out by Zeffren et al., ${ }^{67}$ the exact location of the true fixation point may vary considerably within the foveal avascular zone (FAZ).

Besides the risk of eccentric fixation points, the impact of laser treatment on visual acuity is usually solely attributed to the loss of cone outer segments within the affected tissue column. However, within this column additional pathways associated with the underlying pedicles may be affected (Fig. 7). These synaptic terminals originate from a bundle of cones located more centrally in the fovea. Thus although lesioning of foveolar inner and outer segments could be avoided by direct laser damage, a sector of these cones may still be affected due to lesioning of their displaced terminals. High-resolution assessment of acuity and sensitivity in treated sectors may be able to reveal such effects.

\section{Effects of aging and pathology on the photoreceptor mosaic}

Several studies based on fundus densitometry have indicated loss of visual functions attributed to aging of the photoreceptor mosaic. ${ }^{68,69}$ A specific age-related loss of short wavelength sensitivity may be attributed to a yellowing of the aging lens rather than specific loss of S-cones. ${ }^{70}$ Besides aging, ${ }^{71,72}$ densitometric deficits have also been reported for myopia ${ }^{73}$ and dichromacy. ${ }^{74}$ It is difficult to assess whether the decrease is associated with direct loss of photoreceptors or with other parameters such as alteration of outer segment length or disc packing. Data from monkey retinas ${ }^{75,76}$ suggest that both foveal and peripheral cone densities decrease in advanced age. For human retina Hollyfield ${ }^{77}$ found a correlated loss of rods and ganglion cells while foveal cone (and retinal pigment epithelium) densities remained stable. Similarly Curcio et al. ${ }^{78}$ and PandaJonas et al..$^{71}$ have found that central cone density was not correlated with age while in peripheral retina some decline was notable. It is the rod population that seems to decline continuously, especially in the area of highest density $\left(30^{\circ}\right)$ with a loss of $c$. $30 \%$ during midlife. Beyond this perifoveal region rod numbers appear to remain stable. 
The individual variability of photoreceptor densities within age groups is large and makes it difficult to establish links between specific pathological conditions and receptor densities. Some pathological conditions such as age-related macular degeneration ${ }^{79}$ or retinitis pigmentosa ${ }^{80}$ appear to specifically affect cone subpopulations. In cases of secondary angle-closure glaucoma photoreceptor counts were found to be significantly decreased $^{80}$ and the organisation of the foveal mosaic severely degraded. ${ }^{8,81}$ However, in glaucoma induced experimentally in primates, the cones seemed to remain largely unaffected. ${ }^{82}$

\section{Conclusions}

This short review has pointed to some of the many new aspects of photoreceptor topography revealed by recent studies. Further work on the adult and developing retina will provide more reliable data on the normal condition and variability of the three receptor submosaics. This will improve our ability to detect topographic alterations induced by inherited and acquired pathological conditions.

I thank M. Glösmann and C. Schubert for technical assistance and A. Kafka for helpful suggestions on the manuscript. We are grateful to C. Skorpik and A. Amon (Cornea Transplant Laboratory of the Vienna University Eye Clinic) for the provision of donor tissue. D. Pum has kindly helped with the preparation of some unpublished material.

\section{References}

1. Wandell BA. Foundations of vision. Sunderland, Mass: Sinauer 1995.

2. Yamada E. Some structural features of the fovea centralis in the human retina. Arch Ophthalmol 1969;82:151-9.

3. Misotten L. Estimation of the ratio of cones to neurons in the fovea of the human retina. Invest Ophthalmol Vis Sci 1974;13:1045-9.

4. Adams CK, Perez JM, Hawthorne MN. Rod and cone densities in the Rhesus. Invest Ophthalmol Vis Sci 1974;13:885-8.

5. Østerberg GA. Topography of the layer of rods and cones in the human retina. Acta Ophthalmol (Copenh) 1935;13:1-103

6. Polyak S. The retina. Chicago: University of Chicago Press, 1941.

7. Curcio CA, Hendrickson AE. Organisation and development of the primate photoreceptor mosaic. Prog Retinal Res 1991;10:89-120.

8. Pum D, Ahnelt PK, Grasl M. Iso-orientation areas in the foveal cone mosaic. Vis Neurosci 1990;5:511-23.

9. Chiu MI, Nathans J. Blue cones and cone bipolar cells share transcriptional specificity as determined by expression in human blue visual pigment-derived transgenes. J Neurosci 1994;14:3426-36.

10. Schneeweis DM, Schnapf JL. Photovoltage of rods and cones in the macaque retina. Science 1995;268:1053-6.

11. Nihira M, Anderson K, Gorin FA, Burns MS. Primate rod and cone photoreceptors may differ in glucose accessibility. Invest Ophthalmol Vis Sci 1995;36:1259-70.

12. Ahnelt PK, Keri C, Kolb H. Identification of pedicles of putative blue-sensitive cones in human and primate retina. $\mathrm{J}$ Comp Neurol 1990;293:39-53.

13. Bron AJ, Tripathi RC, Tripathi BJ. Wolff's anatomy of the eye and orbit. 8th ed. London: Chapman and Hall, 1997.
14. Borwein BD, Borwein D, Medeiros J, McGowan JW. The ultrastructure of monkey photoreceptors with special reference to the structure, shape, size and spacing of foveal cones. Am J Anat 1980;159:125-46.

15. Borwein B. Scanning electron microscopy of monkey foveal photoreceptors. Anat Rec 1983;205:363-73.

16. Williams RW. The human retina has a cone enriched rim. Vis Neurosci 1991;6:403-6.

17. Chun MH, Grunert U, Martin PR, Wässle $H$. The synaptic complex of cones in the fovea and in the periphery of the macaque monkey retina. Vision Res 1996;36:3383-95.

18. Hopkins JM, Boycott BB. The cone synapses of DB1 diffuse, DB6 diffuse and invaginating midget, bipolar cells of a primate retina. J Neurocytol 1996;25:381-90.

19. Ahnelt P, Pflug R. Telodendritic contacts of cones in the human fovea. Experientia 1986;42:288-300.

20. Kaiser PK, Boynton RM. Human colour vision. 2nd ed. Washington, DC: Optical Society of America, 1996.

21. Mollon JD, Estévez O, Cavonius CR. The two subsystems of colour vision and their roles in wavelength discrimination. In: Blakemore $C$, editor. Vision: encoding and efficiency. Cambridge: Cambridge University Press, 1990:117-31.

22. Bowmaker J. Evolution of colour vision in vertebrates. Eye 1998;12:541-7.

23. Calkins DJ, Schein SJ, Tsukamoto Y, Sterling P. M and L cones in macaque fovea connect to midget ganglion cells by different numbers of excitatory synapses. Nature 1994;371:70-2.

24. Mollon JD, Bowmaker JK. The spatial arrangement of cones in the primate fovea. Nature 1992;360:677-9.

25. Packer OS, Williams DR, Bensinger DG. Photopigment transmittance imaging of the primate photoreceptor mosaic. J Neurosci 1996;16:2251-60.

26. Ahnelt PK, Kolb H, Pflug R. Identification of a subtype of cone pohtoreceptor, likely to be blue sensitive in the human retina. J Comp Neurol 1987;255:18-34.

27. Curcio CA, Allen KA, Sloan KR, Lerea CL, Hurley JB, Klock IB, Milam AH. Distribution and morphology of human photoreceptors stained with anti-blue opsin. J Comp Neurol 1991;312:610-24.

28. Marc RE, Sperling HG. Chromatic organisation of primate cones. Science 1977;196:454-6.

29. McCrane EP, deMonasterio FM, Schein SJ, Caruso RC. Nonfluorescent dye staining of primate blue cones. Invest Ophthalmol Vis Sci 1983;24:1449-55.

30. deMonasterio FM, McCrane EP, Newlander JK, Schein SJ. Density profile of blue-sensitive cones along the horizontal meridian of macaque retina. Invest Ophthalmol Vis Sci 1985;26:289-302.

31. Nork TM, McCormick SA, Chao GM, Odom JV. Distribution of carbonic anhydrase among human photoreceptors. Invest Ophthalmol Vis Sci 1990;31:1451-8.

32. Diaz-Arraya CM, Provis JM, Billson FA. NADPH-diaphorase histochemistry reveals cone distribution in adult human retina. Aust NZ J Ophthalmol 1993;21:171-9.

33. Szél A, Diamantstein T, Röhlich P. Identification of the bluesensitive cones in the mammalian retina by anti-visual pigment antibody. Comp Neurol 1988;273:593-602.

34. Kolb H, Goede P, Roberts S, McDermott R. Gouras P. Uniqueness of $\mathrm{S}$-cone pedicle in the human retina and consequences for colour processing. J Comp Neurol 1997;386:443-60.

35. Naarendorp F, Rice KS, Sieving PA. Simulation of rod and cone signals at threshold in human observers. Vision Res 1996;36:2681-8.

36. Ahnelt PK, Kolb H. Horizontal cells and cone photoreceptors in human retina: a Golgi-electron microscope study of spectral connectivity. J Comp Neurol 1994;343:406-27.

37. Dacey DM, Lee BB, Stafford DM, Smith VC, Pokorny J. Horizontal cells of the primate retina: cone specificity without cone opponency. Science 1996;271:656-8. 
38. Kouyama N, Marshak DW. Bipolar cells specific for blue cones in the macaque retina. J Neurosci 1992;10:3045-55.

39. Curcio CA, Sloan KR, Packer O, Hendrickson A. Distribution of cones in human and monkey retina: individual variability and radial asymmetry. Science 1987;236:579-82.

40. König A. Über den menschlichen Sehpurpur und seine Bedeutung für das Sehen. Berlin: S B Akad Wiss, 1894:577-98.

41. Willmer EN. Colour of small objects. Nature 1944;153:774-5.

42. Wilmer EN, Wright WD. Colour sensitivity of the fovea centralis. Nature 1945;156:119-121.

43. Williams DR, MacLeod DIA, Hayhoe M. Foveal tritanopia. Vision Res 1981;21:1341-56.

44. Williams DR, MacLeod DIA, Hayhoe M. Punctate sensitivity of the blue-sensitive mechanism. Vision Res 1981;21:1357-75.

45. Röhlich P, Ahnelt PK, Dawson WW, Szél A. Presence of immunoreactive blue cones in the fetal monkey fovea. Exp Eye Res 1994;58:249-52.

46. Schwalbe G. Mikroskopische Anatomie des Sehnerven, der Netzhaut und des Glaskörpers. In Graefe A, Saemisch T, editors. Handbuch der Allgemeinen Augenheilkunde, vol 1. Leipzig: W Engelmann, 1874.

47. MacLeish PR, Wikler KC. Evidence for immature cones in the far periphery of the primate retina [abstract]. Invest Ophthalmol Vis Sci 1997;38:31.

48. Mollon JD, et al. What is the function of the cone-rich rim of the retina. Eye 1998;12:548-52.

49. Fine BS, Yanoff M. Ocular histology: a text and atlas. 2nd ed. Hagerstown: Harper and Row, 1975.

50. Sigelman J, Ozanics V. Retina. In: Jakobiec FA, editor. Ocular anatomy and teratology. Philadelphia: Harper and Row, 1982:441-506.

51. O'Malley PF, Allen RA. Peripheral cystoid degeneration of the retina: incidence and distribution in 1000 autopsy eyes. Arch Ophthalmol 1967;6:769-76.

52. Foos RY, Feman SS. Reticular cystoid degeneration of the peripheral retina. Am J Ophthalmol 1970;69:392-403.

53. Schein SJ. Anatomy of macaque fovea and spatial densities of neurons in foveal representation. J Comp Neurol 1988;269:479-505.

54. Diaz-Araya C, Provis JM. Evidence of photoreceptor migration during early foveal development: a quantitative analysis of human fetal retinae. Vis Neurosci 1992;8:505-14.

55. Yuodelis C, Hendrickson A. A qualitative and quantitative analysis of the human fovea during development. Vision Res 1986;26:847-55.

56. Perry VH, Cowey A. The length of the fibres of Henle in the retina of macaque monkeys: implications for vision. Neuroscience 1988;25:225-36.

57. Leventhal AG, Ault SJ, Vitek DJ. The nasotemporal division in primate retina: the neural bases of macular sparing and splitting. Science 1988;240:66-7.

58. Savy C, Simon A, Nguyen-Legros J. Spatial geometry of the dopamine innervation in the avascular area of the human fovea. Vis Neurosci 1991;7:487-98.

59. Ahnelt PK, Keri C. Cone pedicles in the fovea and periphery of the human retina. Invest Ophthalmol Vis Sci (ARVO Suppl) 1991;32:1128.

60. Haley TL, Pochet R, Baizer L, Burton MD, Crabb JW, Parmentier M, Polans AS. Calbindin D-28K immunoreactivity of human cone cells varies with retinal position. Vis Neurosci 1995;12:301-7.

61. Yellott JI Jr. Spectral analysis of spatial sampling by photoreceptors: topological disorder prevents aliasing. Vision Res 1982;22:1205-10.
62. Curcio CA, Sloan KR. Packing geometry of human cone photoreceptors: variation with eccentricity and evidence for local anisotropy. Vis Neurosci 1992;9:169-80.

63. Sekiguchi N, Williams DR, Packer O. Nonlinear distortion of gratings at the foveal resolution limit. Vision Res 1991;31:815-31.

64. Williams D, Sekiguchi N, Brainard D. Colour, contrast sensitivity, and the cone mosaic. Proc Natl Acad Sci USA 1993;90:9770-7.

65. Marcos S, Tornow R-P, Elsner AE, Navarro R. Foveal cone spacing and cone photopigment density difference: objective measurements in the same subjects. Vision Res 1997;37:1909-15.

66. Bumsted K, Jason C, Szél A, Hendrickson A. Spatial and temporal expression of cone opsins during monkey retinal development. J Comp Neurol 1997;378:117-34.

67. Zeffren BS, Applegate RA, Brodley A, van Heuven WAJ. Retinal fixation point location in the foveal avascular zone. Invest Ophthalmol Vis Sci 1990;31:2099-105.

68. van Norren D, van Meel GJ. Density of human cone photopigments as a function of age. Invest Ophthalmol Vis Sci 1985;26:1014-6.

69. Kilbride PE, Hutman LP, Fishman M, Read JS. Foveal cone pigment density difference in the aging human eye. Vision Res 1986;26:321-5.

70. Haegerstrom-Portnoy G. Short wavelength-sensitive cone sensitivity loss with aging: a protective role for macular pigment? J Opt Soc Am [A] 1988;5:2140-4.

71. Panda-Jonas S, Jonas JB, Jakobczyk-Zmija M. Retinal photoreceptor density decreases with age. Ophthalmology 1995;102:1853-9.

72. Saito A, Miyake Y, Wang JX, Yagasaki K, Matsumoto Y, Horio N, Horiguchi M. Foveal cone densitometry and changes in foveal cone pigments with aging. Nippon Ganka Gakkai Zasshi 1995;99:212-9.

73. Horio N, Miyake Y, Horiguchi M. Foveal cone densitometry in high myopia. Nippon Ganka Gakkai Zasshi 1996;100:2359.

74. Berendschot TT, van de Kraats J, van Norren D. Foveal cone mosaic and visual pigment density in dichromats. J Physiol (Lond) 1996;492:307-14.

75. Packer O, Hendrickson AE, Curcio CA. Developmental redistribution of photoreceptors across the Macaca nemestrina (pigtail macaque) retina. J Comp Neurol 1990;298:472-93.

76. Ordy JM, Brizzee KR, Hansche J. Visual acuity and foveal cone density in the retina of the aged rhesus monkey. Neurobiol Aging 1980;1:133-40.

77. Hollyfield JG. Aging of the human retina: differential loss of neurons and retinal pigment epithelial cells. Invest Ophthalmol Vis Sci 1992;33:1-17.

78. Curcio CA, Leigh Millican C, Allen KA, Kalina RE. Aging of the photoreceptor mosaic: evidence for selective vulnerability of rods in central retina. Invest Ophthalmol Vis Sci 1993;33:3278-96.

79. Curcio CA, Medeiros NE, Leigh Millican C. Photoreceptor loss in age-related macular degeneration. Invest Ophthalmol Vis Sci 1996;37:1236-49.

80. Milam AH, Jacobson SG. Photoreceptor rosettes with blue opsin immunoreactivity in retinitis pigmentosa. Ophthalmology 1990;12:1620-31.

81. Panda S, Jonas JB. Decreased photoreceptor count in human eyes with secondary angle-closure glaucoma. Invest Ophthalmol Vis Sci 1992;33:2532-6.

82. Wynganski T, Desatnik H, Quigley HA, Glovinsky Y. Comparison of ganglion cell loss and cone loss in experimental glaucoma. Am J Ophthalmol 1995;120:184-9. 